\title{
Le langage juridique dans le Français du tourisme (FOS): les conditions générales de vente
}

\section{Legal language in French language for tourism (FOS): the agreement terms \& conditions}

\author{
KHATIMA EL KRIRH \\ Universidad de Cádiz \\ khatimab@yahoo.fr
}

\begin{abstract}
This article aims at the study of the legal language anchored in the Terms ans conditions $(T \& C)$ of the tourist field. Indeed, there is a need to integrate multidisciplinary aspects into the courses of FOS (French Tourism) in order to develop the skills of professional practice among our srudents. The approach of the discursiva approach allows us to become aware of the implication of the macrostructure and the legal concepts in this tectual genre 'hybrid'. In this sense, the Terms ans conditions reprents the cornerstone in any operation of intelligence of tourist services.
\end{abstract}

\section{Keywords}

Tourism, FOS, legal language, contracts.

\begin{abstract}
Resumen
Este trabajo tiene como objetivo el estudio del lenguaje jurídico en el género turístico Condiciones generales de venta (CGV). Existe la necesidad de integrar aspectos multidisciplinarios en los cursos de FOS

(francés turístico) para desarrollar las habilidades de práctica profesional entre nuestros estudiantes. El enfoque discursivo nos permite tomar conciencia de la implicación tanto de la macroestructura como de los términos legales utilizados en este género 'híbrido'. La importancia de este estudio se basa en la importancia de las CGV, piedra angular en cualquier operación de compraventa de servicios turísticos a futuros clientes o intermediarios del sector turístico.
\end{abstract}

Palabras clave

Turismo, FOS, lenguaje jurídico, contratos. 


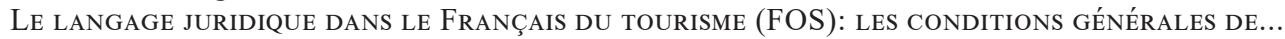

\section{Introduction}

Le tourisme est un secteur vital de l'économie dont le chiffre d'affaires est constamment en hausse ce qui explique que les entreprises de ce secteur recrutent des professionnels multilingues avec d'excellentes compétences communicatives qui leur garantissent une meilleure communication avec leurs clients.

Dans une approche pragmatique concernant la formation de futurs professionnels, et plus précisément dans le domaine touristique, la maîtrise des compétences communicatives dans une ou plusieurs langues étrangères s'avère nécessaire dans le processus d'enseignement-apprentissage notamment du Français sur objectifs spécifiques (désormais FOS). Ainsi, l'examen des objectifs spécifiques requis dans la formation des étudiants en français touristique au niveau universitaire ${ }^{1}$, nous amène à une prise de conscience des difficultés qui entraînent certains textes légaux tels que les Conditions générales de vente fortement impactés par la terminologie juridique. La non-inclusion de ces genres discursifs dans les cours de Français du tourisme entraîne indéniablement une maîtrise insuffisante du Français touristique impliquant par la suite que nos élèves pourraient être confrontés à de vraies difficultés à communiquer avec leurs futurs clients potentiels. Ceci justifie une demande d'élargissement des genres discursifs utilisés dans la didactique du Français touristique.

En effet, notre objectif majeur dans cet ouvrage est de décortiquer les conventions textuelles et le vocabulaire spécialisé, en particulier la terminologie du droit des dits textes afin que les apprenants puissent les reconnaître et les reproduire en tant que professionnels du domaine touristique. Pour ce faire, dans un tout premier temps, nous évoquerons d'abord les nuances du Français de Spécialité et celles du Français sur objectifs spécifiques. Ensuite, les notions de compétences communicatives et de compétence discursive seront abordées du point de vue pragmatique pour délimiter le cadre théorique dans lequel ce travail sera considéré. Dans un deuxième temps, nous proposerons une analyse macro-textuelle du genre discursif dont nous analyserons et classerons la terminologie extraite du corpus proposé à cette fin avant de conclure sur les résultats obtenus et leur incidence dans une conception didactique interdisciplinaire conçue pour l'enseignement-apprentissage du Français touristique.

\section{Français du tourisme, Français sur objectifs spécifiques ou français de spécialité?}

Jean-Marc Mangiante et Chantal Parpette (2004) dans leur ouvrage Le français sur objectif spécifique, de l'analyse des besoins à l'élaboration d'un cours, établissent la distinction entre Français de Spécialité et FOS, qui se tient au fait que ces itinéraires de formation s'inscrivent dans une démarche différente: demande dans le cas du FOS, offre dans le cas du Français de Spécialité (Carras et ali., 2007: 18). Donc, dans le premier on est face à une

1 Niveau intermédiaire de langue française (b1-b2) envisagé pour les deux dernières années du Grado en Turismo pour les cours d'études de tourisme en Espagne. 
demande de formation, qui émane du terrain (institution, université, entreprise) destinée à 'un public précis', clairement identifié, et qui a un lien direct avec un objectif de sortie. Alors qu'il s'agit du Français de Spécialité propre à un domaine professionnel particulier "recouvrant l'ensemble des situations de communication spécialisée propre à une discipline ou à une profession sans connaissance préalable d'un public spécifique auquel s'adresserait un programme de formation linguistique" (Mangiante, 2006: 137-15). Outre, il convient de citer encore d'autres modalités de l'enseignement-apprentissage du FLE, à savoir le Français à visée Professionnelle et le Français Langue Professionnelle. La première vise à faire acquérir des compétences communes à différents secteurs d'activité, à différents postes de travail afin que les apprenants parviennent à combler leurs besoins de communication dans le monde professionnel (Mourlhon-Dallies, 2006) ${ }^{2}$. Or, l'enseignement du Français Langue Professionnelle serait consacré à des publics en voie de spécialisation, en voie de professionnalisation. Ce genre de public apprend un métier en même temps que la langue française. En tenant compte de ces définitions, nous adhérons à l'opinion de (Carras et al., 2007: 20-21) qui reprend les notions de Français à visée Professionnelle et celle de Français Langue Professionnelle pour rappeler qu'elles ne s'opposent pas aux appellations précédentes (FOS et Français de Spécialité), elles les complètent plutôt ${ }^{3}$.

D'un autre côté, en ce qui concerne le Français du tourisme comme discours spécialisé, il faut d'abord préciser que l'évolution du concept de discours spécialisé s'est faite à partir des études de vocabulaire spécialisé appliqué à chaque domaine scientifique et technique. Tel est le cas de certains travaux importants signalés par Lehmann (1988: 90-91 cité par Cantón Rodríguez, 2006: 116), le Vocabulaire d'initiation aux études agronomiques, le vocabulaire d'initiation à la géologie, le vocabulaire Général d'Orientation Scientifique en plus de dictionnaires contextuels qui deviendraient par la suite le trait d'union avec les discours. Toutefois, il nous semble que le débat scientifique contemporain se pose essentiellement sur la distinction ou non, entre langue spécialisé et/ou discours spécialisé sur laquelle Michel Petit (2010) avait fait plusieurs précisions dont la plus intéressante, à notre avis, est celle du point de vue de l'analyse du discours. Dans cette vision, le discours spécialisé est conçu comme un objet langagier complexe issu de plusieurs éléments de nature plus ou moins linguistique, confectionnés par des aspects pragmatiques, lexicaux, morphosyntaxiques et éléments textuels (Gotti, 2003: 11 cité par M. Petit, 2010: 21); caractéristiques 'des usages langagiers propres à l'exercice de certaines activités' (Charaudeau \& Maingueneau, 2002: 540) en renvoyant à des éléments extralinguistiques de contenu cognitif notamment. Par conséquent, les

2 Par exemple, rédiger un $\mathrm{CV}$, un compte rendu, participer à une réunion, avoir un entretien d'embauche etc.

3 Cela démontre que l'enseignement-apprentissage du FLE à ces publics dont les postulats s'attachent à une approche actionnelle, se trouve davantage focalisée sur la réalisation d'actes de paroles, l'accomplissement de tâches ou de projets. Les apprenants sont ainsi devenus des acteurs sociaux (Voir CECR, 2000) dont l'action est motivée par des besoins et des objectifs bien réels (Goulier, 2006: 21). 


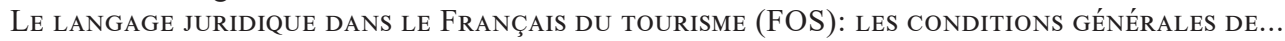

discours produits dans le contexte du Français du tourisme sont divers et proprement liés au domaine touristique.

\section{Approche communicative, compétence discursive et genres discursifs}

Dans le contexte de l'enseignement-apprentissage des langues, celles-ci sont utilisées généralement par les apprenants dans quatre domaines: éducationnels, public, personnel et professionnel sans oublier que ces derniers pourraient converger dans une même situation de communication (CERC, 1990: 18). C'est pour cette raison que nous soutenons qu'une approche pragmatique de la communication et du langage est indispensable du point de vue théorique et pratique. Ainsi, en suivant le CECR, parmi les compétences que l'approche communicative recouvre, nous nous intéressons à la compétence pragmatique axée sur la compétence discursive, la compétence fonctionnelle y compris la conception schématique.

D'une part, la compétence fonctionnelle renvoie à l'utilisation de la langue à des fins fonctionnelles dont le Threshold Level (1990, chapitre 5 cité par Bardière:2016) fournit deux types distinctifs. Le premier concerne les micro-fonctions visant l'utilisation des énoncés simples par les apprenants selon la situation communicative (établir des relations sociales, donner et demander des informations etc.). Le deuxième consacre les macro-fonctions ciblant définir l'utilisation fonctionnelle d'un discours structuré par des phrases ${ }^{4}$. D'autre part, les schémas d'interaction (de la compétence fonctionnelle) visent leur utilisation en se basant sur des modèles d'interaction sociale tels que les modèles d'échange verbaux au regard d'une communication interactive qui comprend des suites structurées d'actions effectuées à tour de rôle par les différentes parties (question/réponse; déclaration/accord, désaccord; requête, offre, excuse/acceptation, refus).

Par ailleurs, la compétence discursive, encadrée bien évidemment dans la compétence pragmatique, est définie par le CECR $(1990,2000)$ comme la connaissance des phrases, de ses composants et la capacité de les maîtriser en termes ainsi que la capacité de gérer, de structurer le discours et le plan du texte. Ici, la connaissance des conventions organisationnelles des textes est acquise par une communauté donnée en se demandant comment:

- l'information est-elle structurée par les macro-fonctions (description, narration, argumentation)

- l'argumentation est-elle construite

- les textes écrits sont-ils mis en page

- la relation entre tâches, stratégies et texte se fait-elle

4 À titre d'exemple: l'argumentation, la description, etc. Autrement dit, la compétence fonctionnelle dans le contexte d'enseignement-apprentissage du FOS, se base sur la réalisation des fonctions précises liée à l'utilisation ultérieure de la langue dans un contexte professionnel. 
En lien avec l'apprentissage du Français touristique, nous pouvons constater alors qu'il s'agit désormais de reconnaître, autant en termes de réception que de production, les genres textuels touristiques dans chaque situation d'interaction professionnelle dont le discours, les conventions textuelles et terminologie se différencient (brochure, guide touristique, dépliant, conditions générales de vente, etc.)

\subsection{Les genres discursifs touristiques}

D'après Beacco (2004), tout locuteur se définit par 'son répertoire discursif comprenant les genres de la communication verbale dont il dispose. Ceux-ci sont présents dans notre communication verbale quotidienne sous forme de petits genres quotidiens" (Bakhtine M.,1929., cité par Beacco, 2013: 12) qui sont échangés aussi dans des communautés restreintes de type professionnel, économique, religieux, sportif, politique, scientifique etc., qui ont été décrits au moyen du terme de topologie discursive (Beacco, 1995: 13-16). De là nous entendons que, dans l'approche discursive ${ }^{5}$, le concept de genre de discours, est incontournable dans l'enseignement/apprentissage des langues sur objectifs spécifiques puisqu'il désigne des formes de communication verbale propres à des communautés discursives (Beacco, 2013). Cet argument se manifeste dans le domaine d'enseignement du FOS notamment du Français touristique dans la mesure où nos élèves feront face à différents genres textuels imposés par leur pratique professionnelle quotidienne dès leur insertion au marché du travail (savoir-faire langagier du CECRL). En amont, il faut rappeler que le débat sur le classement du langage touristique comme un genre discursif a été examiné par plusieurs chercheurs comme Castello (2002), Gotti (2005), Calvi (2000), Gross (1998) et Kelly (1997). Par exemple, pour ces deux derniers auteurs (cités par Martinez Motos:124), la dénomination 'texte touristique 'regroupe un éventail de textes/genres (brochures, guides touristiques, guides de monuments, menus etc.) dont le point commun est le voyageur ou le touriste:

El término 'texto turístico' es un hiperónimo que abarca varias subclases de texto como guías turísticas, catálogos de los grandes operadores, artículos sobre destinos vacacionales en los suplementos de los diarios, folletos de las oficinas de información de turismo, etc. Su denominador común es el destinatario (Martínez Motos, 2005: 124).

Dans le même ordre d'idées, parmi les chercheurs cités ci-dessus, il nous paraît important de mettre l'accent sur le projet Linguaturismo de la professeure Vittoria Calvi (2010:21) qui consiste à créer des corpus textuels touristiques parallèles et comparables en langue espagnole et italienne. Cela fut possible grâce à la taxonomie par 'genre', une perspective qui a été basée sur le travail mené par García Izquierdo et Monzó Nebot (2003) en présentant deux niveaux. L'un est le niveau supérieur qui inclut, d'une optique plus générale,

5 Annoncé par le Cadre européen commun de référence pour les langues (Conseil de 1'Europe, 2001). 
les familles de genres (Calvi, 2013: 14) différenciées dans les discours touristiques: genres éditoriaux, genres institutionnels, genres commerciaux, genres organisateurs, genres légaux, genres scientifique et académique et finalement genres informels. Alors qu'au niveau inférieur nous retrouvons les genres et les sous-genres discursifs. Néanmoins cette nomenclature, Calvi avertit que celle-ci n'est guère définitive en nous rappelant qu'il y a bien d'autres genres touristiques qui sont 'hybrides' comme l'indique Bhatia 'mixed or embedded genre' (cité par Calvi, 2013: 7).

De leur côté, Hatim et Mason (1990: 146) semblent partager la même idée, si bien appliqués aux types de textes. Pour ces auteurs, ceux-ci ne devraient pas être classés avec rigidité sinon qu'on doit prendre en considération les concepts de la multifonctionnalité et le contexte. C'est le cas du genre discursif que nous analysons dans ce travail qui, selon la nomenclature mise en œuvre par Calvi ainsi que sur les diverses affirmations exposées ci-dessus, nous fait constater que le genre Conditions générales de vente, s'encadre non seulement dans les genres légaux mais aussi dans les genres organisateurs du domaine touristique comme nous aurons l'occasion de vérifier au fil des pages suivantes.

\section{Macrostructure et terminologie dans les Conditions générales de vente}

Nous consacrons notre étude aux conditions générales de vente, désormais CGV relevant du droit civil. Le Code civil français définit le contrat comme "une convention par laquelle une ou plusieurs personnes s'obligent, envers une ou plusieurs autres, à donner, à faire ou à ne pas faire quelque chose" (Code civil, art.1101). Il s'agit donc d'un acte juridique basé sur la volonté entre deux ou plusieurs parties ${ }^{6}$.

Les contrats touristiques se distinguent généralement en deux blocs. Le premier englobe ceux de consommation dont le touriste est le destinataire final de la prestation de service; ces derniers pouvant être directs, ou indirects s'il y a une intermédiation entre le prestataire et le consommateur.

Or, le deuxième bloc regroupe les contrats touristiques tenus entre plusieurs entreprises du secteur touristique afin de coordonner leur prestation de services touristiques ${ }^{7}$. Parmi les contrats du premier bloc, on retrouve le $\mathrm{CGV}^{8}$. Celui-ci est un document légal entre

6 Un acte juridique est toute manifestation de volonté destinée à produire des effets de droit, tout acte volontaire spécialement accompli en vue de produire des effets de droit dont la nature et la mesure ont elles-mêmes été voulues par l'auteur de l'acte. C'est non seulement la manifestation de deux volontés concordantes, dont le type même est le contrat, mais ce peut être la manifestation d'une volonté unilatérale.

7 Dénommé aussi comme Contrat de référencement Tour opérateur. Ce contrat va notamment spécifier le taux de commission perçu par le réseau d'agences et peut également comprendre des engagements sur les moyens engagés pour commercialiser le partenaire ou même des engagements de volumes de ventes.

8 En France il est soumis à la réglementation du code du tourisme alors qu'en Espagne n'existe pas un code comme tel mais bien évidemment une réglementation touristique dispersée. Comme tout contrat, le contrat de conditions générales atteste des droits et obligations des parties y souscrites qu'en cas de non-exécution d'une des parties, l'autre aura le droit de dénoncer et d'obtenir un paiement de dommages et intérêts. 
les prestataires et les consommateurs d'un service touristique (soumis au droit privé) tenu au moment d'accomplissement d'une opération de vente (prestataire)/achat (consommateur) dès lors les parties sont engagées à l'honorer. Autrement dit, c'est un document inhérent à toute commercialisation de produits/ services touristiques tant en présentiel qu'en ligne.

Outre l'aspect prononcé de ce type de contrat, les clauses et les conditions sont énoncées unilatéralement par le vendeur/prestataire sans que le consommateur puisse intervenir au moment de sa rédaction puisqu'il est un contrat d'adhésion' (Pérez Abella, 2012: 46). En effet, l'intérêt pour l'analyse de ce type de contrat est, en premier lieu, comme nous l'avons dit auparavant, notre souci de fournir un enseignement-apprentissage pratique à nos étudiants dans les cours du Français touristique. Deuxièmement, concernant les compétences communicatives, le CGV est un document clef, non seulement pour conclure une opération commerciale avec succès mais aussi pour éviter d'éventuels problèmes légaux et réclamations qui pourraient être dûs à:

- une mauvaise communication avec son client,

- une méconnaissance du contenu/terminologie du contrat de vente,

- $\quad$ un manque de notification de ces conditions précontractuelles à ses clients.

Par ailleurs, plus précisément la non-notification des conditions précontractuelles constitue une infraction à la loi car la réglementation touristique impose aux agents et acteurs professionnels touristiques de communiquer l'information précontractuelle au préalable à la formation du contrat:

Le vendeur, ou l'agent de voyage, informe les intéressés par écrit, préalablement à la conclusion du contrat, du contenu des prestations proposées relatives au transport et au séjour, du prix et des modalités de paiement, des conditions d'annulation du contrat ainsi que des conditions de franchissement des frontières (Article L 211-8 du Code français de tourisme).

De cet article ressort que la vente commerciale comporte l'obligation du professionnel de tourisme de renseigner son client sur les termes de son contrat. C'est-à-dire que, dans notre approche didactique et communicationnelle, si nos élèves ne parviennent pas à reconnaître la structure, la terminologie et les concepts juridiques de ce contrat de vente touristique, réaliseront difficilement leur tâche professionnelle convenue avec leur employeur. Ce serait dû, par conséquent, au fait qu'on ne leur aurait transmis que de simples notions théoriques largement incohérentes avec la pratique réelle de leur métier. C'est justement ce dernier argument qui a éveillé notre intérêt d'explorer la structure et la terminologie de ces contrats dans

9 Article 1110 al.2 du code civil: le contrat d'adhésion est “celui dont les conditions générales, soustraites à la négociation, sont déterminées à l'avance par l'une des parties." Ce type de contrat s'oppose au contrat de gré à gré dans lequel les stipulations sont librement négociées entre les parties. 
le but d'une meilleure préparation de nos étudiants à leur avenir professionnel. À ce stade, nous passons à l'analyse du CGV.

\subsection{Corpus et analyse macro-textuel du CGV}

L'approche discursive par genre privilégie l'analyse du texte par des séquences sémantiques, textuelles et pragmatiques qui leur donnent une forme institutionnalisée. Ainsi, les conventions textuelles d'un genre sont repérées par les divergences existantes entre les trois niveaux de l'analyse textuelle: la macrostructure, la superstructure et la microstructure. Le concept de macrostructure a été forgé par Van Dijk (1977; 1978; 1980).

D'après cet auteur, l'organisation du contenu global du texte se réalise par deux types de structures: la macrostructure et la superstructure textuelle. Tout d'abord, la macrostructure est liée à la sémantique du texte au travers des macro-propositions qui garantissent la cohérence. Ensuite, une superstructure est le schéma abstrait qui établit l'ordre global d'un texte composé par une série de catégories dont les possibilités de combinaison sont axées sur des règles conventionnelles bien que ce niveau d'analyse ne soit pas étudié dans cette occasion.

Enfin, la microstructure est le niveau de base du texte concret visant les relations de cohérence et cohésion établies ente les unités qui composent le texte. (Huerta Hurtado y García Nuñez, 2007). Par conséquent, le contrat comme genre discursif présente une macrostructure imposée par les relations intersubjectives et situationnelles et par le but pragmatique de sorte que tous les composants de ce document visent à produire une fin communicative. En effet, la microstructure qui fait l'objet de notre analyse intègre les unités terminologiques et phraséologiques du langage juridique utilisées dans les CGV. Même si ces contrats appartiennent aux genres de textes 'hautement stéréotypés' (Solova, 2013) certains peuvent inclure d'autres clauses en fonction du type de service et/ou produit touristique souscrit (voyage forfaitaire, réservation etc.). Néanmoins, par loi, ces contrats doivent présenter des clauses légales minimales (prix, annulation, cession, etc.) configurant à la fois la macrostructure institutionalisée de ce genre discursif.

Dans ce cadre, il est évident que dans toute démarche d'analyse terminologique le corpus doit être représentatif du genre discursif et également diversifié pour permettre d'établir un aperçu réel de l'analyse visée. C'est pourquoi notre corpus est composé de 7 contrats de Conditions générales de vente issus de trois différents pays (France, Suisse et Canada-Québec) et divers opérateurs francophones dans le but d'avoir une vision plus complète de la structure et la terminologie utilisées: 


\begin{tabular}{|c|c|c|c|c|c|}
\hline $\begin{array}{c}\mathrm{N}^{\mathrm{o}} \\
\text { Texte }\end{array}$ & $\begin{array}{l}\text { Nom } \\
\text { Agence de voyages / } \\
\text { entreprise touristique }\end{array}$ & $\begin{array}{l}\text { Type de ser- } \\
\text { vice engagé }\end{array}$ & $\begin{array}{c}\text { Parties } \\
\text { Engagées }\end{array}$ & Pays & Format \\
\hline 1 & $\begin{array}{c}\text { www.les entreprises } d u \\
\text { voyage. } f r\end{array}$ & $\begin{array}{l}\text { Forfait tou- } \\
\text { ristique }^{10}\end{array}$ & $\begin{array}{l}\text { Le détaillant }{ }^{11} \\
\text { L’organisateur }^{12} \\
\text { Le client }\end{array}$ & France & $\begin{array}{l}\text { Formulaire } \\
\text { PDF }\end{array}$ \\
\hline 2 & $\begin{array}{l}\text { www.Pyrénées-tou- } \\
\text { risme.com }\end{array}$ & $\begin{array}{l}\text { Séjour et } \\
\text { séminaire }\end{array}$ & $\begin{array}{l}\text { Acheteur } \\
\text { Vendeur }\end{array}$ & France & $\begin{array}{c}\text { CGV } \\
\text { En ligne }\end{array}$ \\
\hline 3 & $\begin{array}{c}\text { www.Tourisme-vald- } \\
\text { marne.com }\end{array}$ & $\begin{array}{l}\text { Loisirs et } \\
\text { arts }\end{array}$ & $\begin{array}{l}\text { Agent de réserva- } \\
\text { tion touristique (Co- } \\
\text { mité Départemental } \\
\text { du Tourisme) } \\
\text { - acheteur }\end{array}$ & France & $\begin{array}{c}\text { CGV } \\
\text { En ligne }\end{array}$ \\
\hline 4 & www.visiterlyon.com & $\begin{array}{l}\text { Prestations } \\
\text { diverses }\end{array}$ & $\begin{array}{l}\text { Office de tourisme } \\
\text { Acheteur/client }\end{array}$ & France & $\begin{array}{c}\text { CGV } \\
\text { En ligne }\end{array}$ \\
\hline 5 & $\begin{array}{l}\text { Voyageur du monde. } \\
\text { com }\end{array}$ & $\begin{array}{c}\text { Voyage } \\
\text { personnalisé }\end{array}$ & $\begin{array}{c}\text { Agence de voyages } \\
\text { Acheteur/client }\end{array}$ & Suisse $^{13}$ & $\begin{array}{c}\text { CGV } \\
\text { En ligne }\end{array}$ \\
\hline 6 & www.Lets Travel.com & $\begin{array}{l}\text { Prestations } \\
\text { diverses }\end{array}$ & $\begin{array}{c}\text { Agence de voyages } \\
\text { Acheteur/client }\end{array}$ & Suisse & $\begin{array}{c}\text { CGV } \\
\text { En ligne }\end{array}$ \\
\hline 7 & $\begin{array}{c}\text { www.les voyages de } \\
\text { jeanmarie.com }\end{array}$ & $\begin{array}{l}\text { Route à } \\
\text { vélo }\end{array}$ & $\begin{array}{l}\text { Organisateur des } \\
\text { circuits à vélo } \\
\text { Acheteur/client }\end{array}$ & Québec $^{14}$ & $\begin{array}{c}\text { CGV } \\
\text { En ligne }\end{array}$ \\
\hline
\end{tabular}

Quant à l'analyse macro-textuelle concernant les deux types de contrats de services touristiques (voyages forfaitaires ${ }^{15}$ et $\mathrm{CGV}^{16}$ ), notamment l'examen du contrat de voyages forfaitaires, le corpus révèle que celui-ci, surnommé tout en un, jouit d'une protection légale imminente par rapport à d'autres types de contrats touristiques, voire une prescription de

10 Constitue un forfait touristique la combinaison d'au moins deux types différents de services de voyage aux fins du même voyage ou séjour de vacances, dépassant vingt-quatre heures ou incluant une nuitée. (Article L211-2 du code du tourisme).

11 Un détaillant est un professionnel autre que l'organisateur, qui vend ou offre à la vente des forfaits élaborés par un organisateur ou des services de voyage assurés par un autre professionnel (Article L211-2 du code du tourisme).

12 Un organisateur est un professionnel qui élabore des forfaits touristiques et les vend ou les offre à la vente, directement ou par l'intermédiaire d'un autre professionnel ou encore conjointement avec un autre professionnel, ou un professionnel qui transmet les données du voyageur à un autre professionnel conformément au e du $2^{\circ} \mathrm{du}$ A du II. Article L211-2.4 du code du tourisme).

13 Les CGV sont prévues dans les lois suisses sur le e-commerce notamment (LCD; RS 241) et l'ordonnance sur l'indication des prix (OIP; RS 942.211).

14 Loi sur les agentes de voyages, chapitre A-10.

15 Code du tourisme français - Article L211-1.

16 Conformément à l'article R.211-12 du Code du tourisme, les conditions générales de vente sont issues des articles R.211-3 à R.211-11 du même code. 
contenu obligatoire puisqu'il s'agit de plusieurs intermédiaires commerciaux à part le client (transport terrestre, logement, vols etc.). La macrostructure du contrat de voyage forfaitaire est conjuguée par les macropropositions suivantes:

- Identification des parties (le détaillant et l'organisateur, les voyageurs, coordonnées du client et autres participants, Information voyage)

- Le prix et annulation

- $\quad$ Formalités (formalités - police; Formalités sanitaire; Mineurs; Formalités spécifiques)

- Contact - responsabilité - réclamations

- $\quad$ Signature (le client; le détaillant)

En revenant sur les conditions générales de vente (CGV) ayant le caractère d'un contrat d'adhésion, le client n'intervient pas au moment de la formation de ces conditions comme nous l'avons déjà souligné ${ }^{17}$. Ce sont des clauses rédigées unilatéralement par le vendeur et adressées à son client qui doit les lire avant de faire son achat ou réservation en ligne, la plupart des contrats de $\mathrm{CGV}$ introduisent les macro-propositions/clauses suivantes ${ }^{18}$ :

Prix

Article R.211-8

Lorsque le contrat comporte une possibilité expresse de révision du prix, dans les limites prévues à l'article L. 211-12, il doit mentionner les modalités précises de calcul, tant à la hausse qu'à la baisse, des variations des prix, et notamment le montant des frais de transport et taxes y afférentes, la ou les devises qui peuvent avoir une incidence sur le prix du voyage ou du séjour, la part du prix à laquelle s'applique la variation, le cours de la ou des devises retenu comme référence lors de l'établissement du prix figurant au contrat (extrait du CGV de Pyrénnés-tourisme.fr).

\section{Transport}

Dans le cas où la compagnie aérienne qui doit assurer votre vol ne serait pas en capacité de vous acheminer, malgré son obligation d'assistance Voyageurs du Monde pourra avoir recours à une autre compagnie afin de vous faire voyager dans des conditions de transport comparables.

Les procédures d'enregistrement à distance (avant de se rendre à l'aéroport) pour obtenir la carte d'embarquement (avec le choix ou non du siège en cabine) sont ré-

17 Voir note en bas de page $\mathrm{n}^{\circ} 8$.

18 La signature dans les contrats conventionnels s'est remplacée par la possibilité de marquer l'option 'accepter' avant de finir la transaction en ligne. Ainsi, le vocabulaire de l'hébergement, hôtellerie et restauration est présent dans tous ces contrats. 
alisables dans les délais fixés par chaque compagnie aérienne en fonction des vols (Extrait du Voyageurdumonde.fr).

\section{Responsabilité civile}

\section{Article 2 - responsabilité.}

Dans les termes de l'article 1211-17 du code du tourisme, le comité départemental du tourisme est responsable dans les termes de l'article 23 de la loi du 13 juillet 1992, qui stipule: toute personne physique ou morale qui se livre aux opérations mentionnées à l'article 1er est responsable de plein droit à l'égard de l'acheteur de la bonne exécution des obligations résultant du contrat, que ces obligations soient à exécuter par elle-même ou par d'autres prestataires de services, sans préjudice de son droit de recours contre ceux-ci. Toutefois elle peut s'exonérer de tout ou partie de sa responsabilité en apportant la preuve que l'inexécution du contrat est imputable soit à l'acheteur, soit au fait, imprévisible et insurmontable, d'un tiers étranger à la fourniture des prestations prévues au contrat, soit à un cas de force majeure (extrait du CGV de tourisme-valdemarne.com).

\section{Annulation et modification}

\section{Article 9 - Annulation/modification du fait du client.}

Toutes les demandes d'annulation doivent clairement faire mention du numéro de réservation, nom et coordonnées des personnes concernées par l'annulation. Est considérée comme une annulation partielle l'annulation par le client d'une partie de la prestation commandée. Toute demande d'annulation sera retenue uniquement sur déclaration faite impérativement par courrier auprès d'Only Lyon Tourisme et Congrès et non auprès des prestataires touristiques (extrait de visiterlyon.com).

\section{Assurance}

Art. 16 - Assurance responsabilité civile.

Une assurance est souscrite à titre principal auprès de la compagnie Allianz (1, cours Michelet-CS 305192076 Paris la défense), couvrant la Responsabilité Civile Professionnelle (RCP), notamment les dommages corporels, matériels et immatériels confondus à hauteur de 1.000 .000 d'euros par année d'assurance.

La notion de responsabilité civile (accident, incendie, vol, autres) varie suivant la législation des pays concernés: il est conseillé aux voyageurs de se garantir individuellement par une assurance complémentaire à celles éventuellement achetées par notre intermédiaire [...]. Pyrénées Tourisme informe le Client de l'existence de contrats d'assurances couvrant notamment les conséquences de l'annulation, précision faite que les assurances ne sont jamais remboursables dans le cadre d'une Commande (extrait du www.pyrenees-tourisme.fr). 


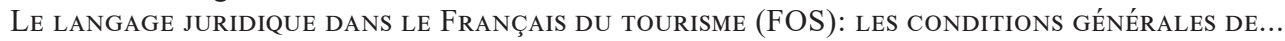

Formalités douanières et administratives.

Passeport, visas et vaccins:

Vous êtes seul responsable d'être en possession des documents nécessaires à votre voyage. Veuillez vous renseigner auprès de votre agence de voyages lors de votre inscription. L'organisateur n'a aucune responsabilité lorsqu'un passager ne se conforme pas à ces obligations. Si vous manquez votre vol (passeport non valable, etc.), vous perdez tous vos droits de transport et n'avez aucun droit à une indemnité ou un remboursement (extrait du Letstravel.com).

Cession du contrat.

Art. 4 - Cession du contrat

Le(s) cédant(s) doit impérativement informer Pyrénées Tourisme de la cession du contrat par lettre recommandée avec accusé de réception au plus tard 7 jours avant le début du voyage (15 jours pour une croisière), en indiquant précisément le(s) nom(s) et adresse du/des cessionnaire(s) et des participant(s) au voyage et en justifiant que ceux- ci remplissent les mêmes conditions que lui pour effectuer le voyage.

4.1. Frais de cession sur un séjour sans transport ou en transport autocar: $15 €$ par personne

4.2. Frais de cession sur séjours incluant un transport par vol charter (extrait du Pyrénées-tourisme-com).

Droit applicable et for juridique.

Art. 18 - Dispositions finales.

Le fait que Pyrénées Tourisme ne se prévale pas, à un moment ou à un autre, d'une des dispositions des présentes Conditions de Vente ne pourra être interprété comme valant renonciation par Pyrénées Tourisme à s'en prévaloir ultérieurement.

Dans le cas où l'une des dispositions des Conditions de Vente serait déclarée nulle ou sans effet, elle serait réputée non écrite, sans que cela n'affecte la validité des autres dispositions, sauf si la disposition déclarée nulle ou sans effet était essentielle et déterminante.

Le client reconnaît être informé qu'en application de l'article L. 121-21-8-12 du code de la consommation, l'ensemble des prestations proposées par Pyrénées Tourisme n'est pas soumis à l'application du droit de rétractation prévu aux articles L. 121-21 et suivants du Code de la consommation en matière de vente à distance (extrait du Pyrénées-tourisme-com). 


\subsection{Le repérage des unités terminologiques dans le CGV}

Nous sommes conscients du fait que la compétence pragmatique entraine incontestablement la familiarisation de nos élèves autant avec le vocabulaire touristique qu'avec le langage juridique utilisés dans ces contrats. Cette familiarisation s'exerce au niveau de la microstructure où l'on retrouve de nombreux termes, expressions voire des phrases stéréotypées dans le langage du droit que nos élèves doivent maîtriser. Sur ce point-là, le discours juridique notamment par le biais des termes juridiques, assure la cohérence et la cohésion de tous les composants macro-propositionnels de ce genre discursif, étant donné qu'il s'agit d'une série de droits et devoirs entre plusieurs intervenants (voyageurs, détaillant, organisateur, client etc.). Par conséquent, le genre CGV est considéré comme un discours juridique dans la mesure où il vise à ce que son contenu soit efficace juridiquement entre les parties y souscrites. Cornu (2007: 22) affirme que 'discours juridique' est “ tout discours qui a pour objet la création ou la réalisation du droit" puisqu'il crée des effets de droit, c'est-à-dire, le caractère juridique d'un texte est dans son but de communication et non pas dans le sujet de communication

Tenant compte de la dimension de cet ouvrage, nous proposons une exploration terminologique du corpus non exhaustive mais qualitative des unités terminologiques que chaque macro-proposition contient. Premièrement, même si le discours appréhendé est juridique, nous constatons que les diverses collocations terminologiques du prix correspondent principalement au domaine économique:

Etablissement du devis - prix en vigueur - prix calculés de manière forfaitaire -tarifs établis - remboursement-prestations incluses dans le prix - taxes d'aéroport-frais de visas - frais supplémentaires - révisions des prix - prix à la hausse/à la baisse - calcul hors taxes - rachat du billet - redevances et taxes afférentes aux prestations offertes - les taxes d'atterrissage/d'embarquement et/ou de débarquement - taux de change-variation répercutée au prorata - frais d'annulation-acompte et paiement du solde - versement-règlement intégral du prix - frais de réservation - frais de dossier - plancher à/plafond à - diminution de prix en déduction des sommes - [trop-perçu] - sans supplément de prix - avance de fond - frais de recherche et de premier secours - modifications du cours de change et de la taxe sur la valeur ajoutée - dépôt additionnel - le solde de la prestation convenue et restant due - un bon d'échange - les retenues - prix (ce qui comprend, ce qui ne comprend pas, prix total TTC, acompte versé, mode de règlement, calendrier de paiement) - révision du prix (part du transport redevances et taxes connues à la date du contrat/part des achats en devises et taux de référence). 
Deuxièmement, la terminologie du droit civil contractuel emprunte les termes de cession du contrat:

Cédant - cessionnaire - frais de cession - frais de cession spécifique - cédant et le cessionnaire sont solidairement responsables du paiement - sauf stipulation plus favorable au cédant - La réservation devient ferme - Préalablement à la conclusion du contrat - des éléments essentiels du contrat - un avenant au contrat.

La même remarque régit les clauses d'annulation et modification:

Annulation et modification du fait du client/du fait de l'organisateur - délais de règlement imposés par les prestataires - toute annulation entraîne la facturation des frais - frais complémentaires sur annulation partielle - frais de modification affectant le programme - modification sur vol charter/vol régulier - donner lieu à des indemnisations et remboursement - circonstances de force majeure - latitude pour modifier l'itinéraire prévu - inscription tardive - être modifiées avant la conclusion du contrat de voyage, modifications éventuelles susceptibles d'être apportées-des frais occasionnés par - l'annulation émanant du client.

Également, la phraséologie utilisée pour la responsabilité des intervenants s'encadre dans le Droit civil contractuel:

Ne savoir être tenu pour responsable de - prestation ne répond pas aux spécifications contractuelles-saisir sans délai un représentant local - s'engager vis-à-vis du client - ne savoir engager la responsabilité de - les éléments contractuels de la réservation appréciation d'ordre subjectif ou personnel - à défaut de réponse satisfaisante dans un délai de- le client peut saisir le médiateur du tourisme et du voyage - agir en qualité d'intermédiaire auprès de - faire valoir toutes revendications justifiées - demande en dommages et intérêts - responsabilité plus étendue - en cas de verbalisation - prise en charge-ne saurait voir sa responsabilité se substituer à celle des transporteurs - nous recommandons fortement de ne pas prévoir d'obligations professionnelles - ne saurait garantir la possibilité de - sur demande expresse du client - il est de la responsabilité du client de - en apportant la preuve de l'inexécution ou de la mauvaise exécution du contrat - no show' - aucun remboursement sera octroyé - l'inobservation par le client de ses obligations - infliger le paiement d'une amende - droit d'accès et de rectification - le vendeur ne se soit réservé expressément le droit de - clause de résiliation et de remboursement sans pénalités - annulation par le voyageur (absence de droit de rétraction, frais d'annulation ou résolution du contrat, barème d'annulation - annulation par le détaillant ou l'organisateur. 
Pourtant, la terminologie localisée au niveau du transport appartient au Droit commercial/des affaires:

Partenaires autocaristes-suppléments demandés - programmes avec transport aérien - responsabilité des transporteurs aériens-conséquences des accidents/incidents sont régies par les dispositions de - les réglementations locales régissant les transports nationaux - vols affrétés et vols réguliers - se réserver le droit de - acheminer les participants par voie terrestre - le cas de pré et post-acheminement émis sur un même billet-transporteurs contractuels ou de fait -feront l'objet d'un supplément à régler - le client sera obligé d'acheter à ses frais-la non-utilisation totale ou partielle du titre de transport - des taxes dont l'exigibilité procède de l'embarquement effectif du passager-refus d'embarquement ou confiscation d'objet jugé dangereux-tout bagage enregistré égaré ou endommagé - déclaration de la part du client auprès de - effectuer une Déclaration Spéciale d'Intérêt - vols avec arrangement terrestre et arrangement terrestre seul - vols sur contingents - est responsable de plein Droit, sans préjudice de son droit de recours-cession commerciale - Transport: Air (vol régulier, vol spécial; détail des plans de vols aller/retour), Fer, Mer ou autre (classe, départ, ville, départ/ retour, date, horaire, durée et lieu des escales);Itinéraire (circuit, croisière) - le voyage de substitution-jour férié /jour ouvrable.

En ce qui concerne les formalités administratives et douanières, les termes s'inscrivent dans le domaine administratif:

Modifications administratives ou sanitaires sont susceptibles d'intervenir - un mineur non accompagné serait inscrit (à son insu) - il est conseillé de se munir de la carte européenne d'assurance - personnes placées sous tutelle ou curatelle -faire état de leur placement - fournir une autorisation écrite de leur curateur-une personne désignée par le juge des tutelles - bulletin d'inscription.

Le droit commercial comprend aussi les assurances souscrites par les parties en application du Droit civil contractuel:

Assurance est souscrite à titre principal auprès de - couvrant la Responsabilité Civile Professionnelle - les dommages corporels - matériels et immatériels confondus à hauteur de-garantir individuellement par une assurance complémentaire à celles éventuellement acheté aucune assurance n'est comprise dans le prix du voyage - ne se prévale pas d'une des dispositions - ne pourra être interprété comme valant renonciation - dispositions des Conditions de Vente - être déclarée nulle ou sans effet - réputée 


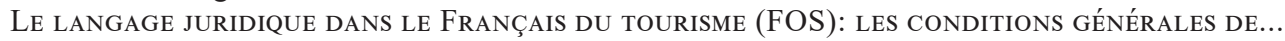

non écrite - client reconnaît être informé qu'en application de l'article xxx - soumis à l'application du droit de rétractation prévu aux articles -, sans préjuger des recours en réparation des dommages - la souscription facultative d'un contrat d'assurance-dommages éventuellement subis - assurance annulation et rapatriement-assurance (proposé par le vendeur et acceptés par le client: multirisque, rapatriement, annulation, assureur, $\mathrm{n}^{\mathrm{o}}$ de police).

Le corpus atteste du maniement des termes relatifs au for juridique, la réglementation et/ ou la juridiction à laquelle les parties doivent se soumettre en cas de litige:

Code de la consommation en matière de vente à distance - une convention de mandat Conformément à l'article - Comité Départemental du Tourisme - se reporter à l'article xxxx du Code du Tourisme - Comité Départemental du Tourisme - la réglementation ou aux usages de pays d'accueil - prévues par le contrat en application de l'article xxxx - Les dispositions du présent article - la conclusion d'un accord amiable - Ombudsman (suisse).

Finalement, en tenant compte du fait que l'objet du CGV est un service touristique, le vocabulaire de l'hôtellerie et restauration est largement étendu:

Intitulé du forfait (type, $\mathrm{n}^{\mathrm{o}}$ de référence, la durée) - pays de destination -hébergement (situation et caractéristiques, catégorie selon les normes locales - langue des animations - nombre de nuits-repas (sans repas, petit déjeuner, demi-pension, pension complète) -all inclusive-activités incluses dans le forfait-personnes à mobilité réduite etc.

\section{Conclusion}

Malgré l'importance du classement par genres dans les études menées sur le Français du tourisme, aucun antécédent d'étude avait visé l'apprentissage-enseignement du langage juridique dans les genres textuels touristiques. Nous avons pu vérifier que la diversité des genres textuels dans le discours touristique ainsi que la conjonction des sous-langages dans un même genre discursif, tel est le cas de $\mathrm{CGV}$, conduisent la pratique pédagogique à cibler l'approche discursive pour mieux appréhender ce type de discours spécialisé.

D'un côté, nous avons constaté que l'analyse macro-textuelle de ce genre discursif atteste d'une 'stéréotypie ' bondée de macropropositions qui assurent la cohérence et cohésion de ce genre textuel comme 'prix', responsabilité', 'transport', juridique, etc. D'un autre côté, l'exploration terminologique du corpus montre que c'est un genre discursif ' hybride ' dans la mesure où il rassemble des sous-langage d'autres spécialités comme l'économie et 
la finance (Prix), le vocabulaire touristique concernant les établissements touristiques et leur équipement (restauration et hôtellerie) ainsi que des termes du droit commercial utilisés dans les clauses du 'transport et 'responsabilité civile et assurance'. De même, dans une perspective juridique, les Conditions générales de vente est un acte juridique soumis à une juridiction déterminée unilatéralement par le vendeur du service. Cela veut dire que les apprenants doivent accorder une attention particulière au système juridique où le contrat prendra effet, dans le but de reconnaître les institutions juridiques compétentes en cas de litige.

Finalement, nous en tirons comme conclusion que l'approche discursive dans l'enseignement d'une langue étrangère, notamment le français du tourisme est bel et bien indispensable pour l'acquisition des compétences communicationnelles d'un point de vue pragmatique. Par conséquent, une bonne maîtrise du genre discursif CGV, non seulement à l'oral mais aussi à l'écrit témoigne de l'engagement qu'ont les enseignants envers leurs apprenants afin de les préparer à surmonter les défis de la communication professionnelle touristique en langue française.

\section{Références bibliographiques}

AdAm, Jean-Michel. 2016. 'La linguistique textuelle et l'analyse de discours, dans le contexte des années 70" in Pratiques, 169-170 [consulté le 23/04/2019] <http://journals.openedition. $\mathrm{org} /$ pratiques/2931; DOI:10.4000/pratiques.2931>.

BARDiÈre, Yves. 2016. “De la pragmatique à la compétence pragmatique " in Recherches en didactique des langues et des cultures [consulté le 24/03/2019] < http://journals.openedition. org $>$.

BeACCO, Jean-Claude. 2013. ' L'approche par genres discursifs dans l'enseignement du français langue étrangère et langue de scolarisation" in Pratiques, 189-200 [consulté le 19/03/2019] < https://journals.openedition.org/pratiques/3838\#toctoln2>.

Beacco, Jean Claude, Simon bouquet \& Remy Porquier, R. 2004. Niveau B2 pour le français/un référentiel. Paris, Hatier.

BeACCO, Jean-Claude. 1995. “À propos de la structuration des communautés discursives: beaux-arts et appréciatif ' in Les Carnets du Cediscor, 3, 136-153[consulté le 10/3/2019] $<\mathrm{http}$ ://journals.openedition.org/cediscor/523>.

Benveniste, Émile. 1966 [1964]. “Les niveaux de l'analyse linguistique” in Proceedings of the Ninth International Congress of Linguists, La Haye.

Calvi, María Vittoria. 2010."Los géneros discursivos en la lengua del turismo: una propuesta de clasificación " in Ibérica, no 19, 9-31.

Cantón Rodríguez, Ma Loreto. 2006. "Les écrits touristiques en cours universitaire de tourisme: typologie textuelle et discursive " in CAUCE Revista Internacional de Filología y su Didáctica, no 29, 111-136 [consulté le 19/03/2019]. <www.centrocervantesvirtual.com>. 
Anales de Filología Francesa, n. ${ }^{\circ}$ 27, 2019

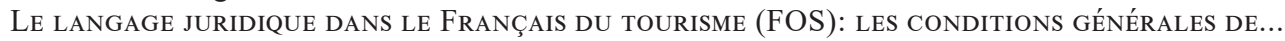

Carras Catherine, Jacqueline tholas, Patricia Kohler \& Elisabeth Szilagyi. 2007. Le français sur objectifs spécifiques et la classe de langue. Paris, Cle International.

Code DU TOURISME. France [consulté le 14 /06/2019] <https://www.legifrance.gouv.fr/> .

Conseil de L'Europe. 2001. Cadre européen commun de référence pour les langues: apprendre, enseigner, évaluer [consulté le 20/02/2019]

Conseil DE L'Europe. Threshold 1990. [consulté le 15/03/2019] <www.ealta.eu.org/documents/resources/Threshold-Level_CUP.pdf $>$.

Charaudeau, Patrick. 2005. "La sémantique de la langue, sémantique du discours". Actes du colloque en hommage à Bernard Pottier [consulté le 15/03/2019] < http://www.patrick-charaudeau.com/Semantique-de-la-langue-semantique.htm $>$

Dorata-ZaróD, Anna. 2005. 'La langue spécialisée comme véhicule d'enseignement de savoir-faire professionnel” in Synergies Pologne No2: L'Europe des langues et des cultures, Tome I: Didactologie des langues - cultures, 163-168.

Goulier, Francis. 2006. Les Outils du Conseil de l'Europe en classe de langue. Paris, Didier.

Huerta Hurtado, María de Jesús et García NúÑez, Roberto. 2007. “Lingüística de texto (superestructuras)" in Sincronía, primavera [consulté le 15/03/2019] <https://sincronia. cucsh.udg.mx/garciaspring07.htm>.

LoIS SUR LE E-COMMERCE. Suisse [consulté le 14 /06/2019] <https://www.kmu.admin.ch/ $\mathrm{kmu} / \mathrm{fr} / \mathrm{home}>$

Loi SUR LES AGENTES DE VOYAGES. Québec [consulté le 14 /06/2019].<http://legisquebec. gouv.qc.ca/fr/ >.

Mangiante, Jean-Marc .2006. “Français de spécialité ou français sur objectif spécifique: deux démarches didactiques distinctes" in Linguistique plurielle: Congreso Internacional de Lingüística Francesa, Valencia,Vol.1,137-15 [consulté le 18/03/2019] < https://dialnet.unirioja.es/servlet/articulo? codigo $=4030419>$.

Mangiante, Jean-Marc \& Chantal Parpette. 2004. “Le Français sur Objectif Spécifique: de l'analyse des besoins à l'élaboration d'un cours ". Paris, Hachette, coll. "F".

Martínez Motos, Raquel. 2005. “Análisis comparativo de las convenciones textuales de los textos turísticos en inglés y en español: los folletos editados por organismos oficiales " in Fuentes Luque, Adrián (éd.). La traducción en el sector turístico. Granada, Atrio, 124.

Mourlhon-Dallies, Florence. 2006. 'Penser le français langue professionnelle ' in Le français dans le Monde, $\mathrm{n}^{\circ} 346,25-28$.

Mourlhon-Dallies, Florence. 2011. "Le français sur objectifs universitaires ', in Celesthia Cediscor, 135-145 [consulté le 10/03/2019]. <https://gerflint.fr/Base/Monde8-T1/mourlhon-dallies.pdf>

Normand, Claudine. 1996. 'Emile Benveniste: quelle sémantique?' in Linx, nº, 221-245 [consulté le 03/02/2019].< http://journals.openedition.org/linx/1183>. 
Normand, Claudine.1992. "Linguistique saussurienne et signification” " in $\operatorname{Linx}, \mathrm{n}^{\circ} 26,49-75$ [consulté le 23/03/2019].<ww.persee.fr/doc/linx_0246-8743_1992_num_26_1_1237>.

PÉrez Abella, Víctor. 2012. Contratos de entrenamiento y tiempo libre: el contrato de viaje combinado con actividades deportivas y de riesgo. DEA. Universidad Complutense de Madrid.

Petit, Michel. 2010. 'Le discours spécialisé et le spécialisé du discours: repères pour l'analyse du discours en anglais de spécialité "in E-rea. [consulté le $23 / 03 / 2019$ ]<http://journals. openedition.org/erea/1400>.

Solová, Regina. 2013. Traduction des genres textuels stéréotypés. Thèse doctorale. Université of Wroclaw.

\section{Corpus}

Texte 1. < https://www.snavmed.org/wp./Contrat-de-voyage > [consulté le 14 /06/2019].

Texte 2. < https://www.pyrenees-tourisme.fr/cgv/> [consulté le 14/06/2019].

Texte 3. < https://www.tourisme-valdemarne.com/> [consulté le 14 /06/2019].

Texte 4. < https://www.visiterlyon.com/conditions-generales-des-ventes/> [consulté le 14 /06/2019].

Texte 5. < https://www.voyageursdumonde.fr/> [consulté le 14/06/2019].

Texte 6. <https://www.letstravel.ch/lets-travel-et-vous/> [consulté le 14/06/2019].

Texte 7. <http://www.lesvoyagesdejeanmarie.com/qui-sommes-nous/> [consulté le 14 /06/2019]. 
